\title{
Rank Selection and Depth Conditions for Balanced Simplicial Complexes
}

\author{
Brent Holmes Justin Lyle \\ Department of Mathematics \\ University of Kansas \\ Kansas, U.S.A. \\ brentjholmes323@gmail.com justin.lyle@ku.edu
}

Submitted: Jan 17, 2020; Accepted: Mar 2, 2021; Published: May 21, 2021

(C) The authors. Released under the CC BY-ND license (International 4.0).

\begin{abstract}
We prove some new rank selection theorems for balanced simplicial complexes. Specifically, we prove that if a balanced simplicial complex satisfies Serre's condition $\left(S_{\ell}\right)$ then so do all of its rank selected subcomplexes. We also provide a formula for the depth of a balanced simplicial complex in terms of reduced homologies of its rank selected subcomplexes. By passing to a barycentric subdivision, our results give information about Serre's condition and the depth of any simplicial complex. Our results extend rank selection theorems for depth proved by Stanley, Munkres, and Hibi.
\end{abstract}

Mathematics Subject Classifications: 05E45, 05E40, 13F55

\section{Introduction}

Let $k$ be a field, $A=k\left[x_{1}, \ldots, x_{n}\right]$, and $I$ a square-free monomial ideal in $A$. The Stanley-Reisner correspondence associates to $R:=A / I$ a simplicial complex $\Delta$ whose topological and combinatorial properties capture the algebraic structure of $R$. Exploiting this correspondence has been an active line of investigation over the past few decades. Due to their combinatorial characterization ([Rei76, Theorem 1]), Stanley-Reisner rings that are Cohen-Macaulay have received particular attention. However, the Cohen-Macaulay property is quite strong in this setting, and so there has been a focus in recent years on considering weaker algebraic properties such as Serre's condition $\left(S_{\ell}\right)$ or bounds on depth $R$ which still have interesting combinatorial ramifications. For instance, even $\left(S_{2}\right)$ forces $\Delta$ to be pure, and $\left(S_{\ell}\right)$ implies the $h$-vector of $R$ is nonnegative up to the $\ell$ th spot ([MT09]); see [PSFTY14] for a survey of related results. The main purpose of this paper is to consider Serre's condition and the depth of Stanley-Reisner rings by studying balanced simplicial complexes. 
A balanced simplicial complex $\Delta$ is a simplicial complex of dimension $d-1$, together with an ordered partition $\pi=\left(V_{1}, \ldots, V_{d}\right)$ of the vertex set of $\Delta$ such that $\left|F \cap V_{i}\right| \leqslant 1$ for every $F \in \Delta$ and every $i$. To put it another way, the vertices of $\Delta$ are colored so that no face of $\Delta$ has more than one vertex of a given color. The motivating example of a balanced simplicial complex is the order complex $\mathcal{O}(P)$ of a finite poset $P$, whose vertex set is $P$ and whose faces consist of all chains in $P$; we partition the vertices of $\mathcal{O}(P)$ by their height in $P$. When $P$ is the face poset of a simplicial complex $\Delta$ (excluding the empty face), $\mathcal{O}(P)$ is nothing but the barycentric subdivision of $\Delta$, and it's well known that its geometric realization is homeomorphic to that of $\Delta$. Thus we can study topological characteristics of any simplicial complex via the combinatorial structure of a balanced simplicial complex. In particular, we may study homological properties such as the Cohen-Macaulay property and Serre's condition $\left(S_{\ell}\right)$, and numerical invariants such as depth in this manner.

Let $(\Delta, \pi)$ be a balanced simplicial complex of dimension $d-1$ with ordered partition $\pi=\left(V_{1}, \ldots, V_{d}\right)$, and let $k[\Delta]$ denote its Stanley-Reisner ring over the field $k$. If $S \subseteq[d]$, we let $\Delta_{S}$ be the subcomplex of $\Delta$ induced on $\bigcup_{i \in S} V_{i}$, and we refer to $\Delta_{S}$ as the $S$-rank selected subcomplex of $\Delta$. It's often convenient to think about the ranks we remove rather than those we retain, and so we also set $\widetilde{\Delta}_{S}:=\Delta_{[d]-S}$. If $S=\{i\}$ is a singleton, we abuse notation and write $\Delta_{i}$ or $\widetilde{\Delta}_{i}$, as appropriate. The so-called rank selection theorems of Stanley ([Sta79]) and Munkres ([Mun84]) show that homological properties often pass from $\Delta$ to $\Delta_{S}$. Specifically, we have the following:

Theorem 1 ([Sta79]). Let $(\Delta, \pi)$ be a balanced simplicial complex. If $k[\Delta]$ is CohenMacaulay, then $k\left[\Delta_{S}\right]$ is Cohen-Macaulay for any $S \subseteq[d]$.

Theorem 2 ([Mun84]). Let $(\Delta, \pi)$ be a balanced simplicial complex. Then, for any $i \in[d]$, $\operatorname{depth} k\left[\widetilde{\Delta}_{i}\right] \geqslant \operatorname{depth} k[\Delta]-1$.

As Serre's condition $\left(S_{\ell}\right)$ generalizes the Cohen-Macaulay property, it is natural to consider if there is any extension of Theorem 1 to $\left(S_{\ell}\right)$. We prove this is indeed the case.

Theorem A. Let $(\Delta, \pi)$ be a balanced simplicial complex of dimension $d-1$. If $k[\Delta]$ satisfies Serre's condition $\left(S_{\ell}\right)$, then $k\left[\Delta_{S}\right]$ satisfies $\left(S_{\ell}\right)$ for any $S \subseteq[d]$.

If $P$ is a finite poset, we let $P_{>j}$ be the subposet consisting of the elements of $P$ with height greater than $j$. In the case $\Delta=\mathcal{O}(P)$ for a finite poset $P, \mathcal{O}\left(P_{>j}\right)$ is the subcomplex of $\Delta$ with the bottom $j+1$ ranks removed. For this case, we have the following refinment of Theorem A.

Theorem B. Let $P$ be a finite poset.

1. If $k[\mathcal{O}(P)]$ satisfies $\left(S_{\ell}\right)$, then $\widetilde{H}_{i-1}\left(\mathcal{O}\left(P_{>j}\right) ; k\right)=0$ whenever $i+j<d$ and $0 \leqslant i<\ell$.

2. If $P$ is the face poset of a simplicial complex $\Delta$ and $\widetilde{H}_{i-1}\left(\mathcal{O}\left(P_{>j}\right) ; k\right)=0$ whenever $i+j<d$ and $0 \leqslant i \leqslant \ell$, then $k[\mathcal{O}(P)]$, and thus $k[\Delta]$, satisfies $\left(S_{\ell}\right)$. 
Thereom $\mathrm{B}$ shows, if $\Delta$ is a simplicial complex and $P$ its face poset, that the reduced homologies $\widetilde{H}_{i-1}\left(\mathcal{O}\left(P_{>j}\right) ; k\right)$ pin the largest $\ell$ for which $\Delta$ satisfies $\left(S_{\ell}\right)$ to two possible values. It's natural to ask whether one can fully characterize $\left(S_{\ell}\right)$ in this way i.e., whether the converse of (1) or (2) hold. We provide examples (Examples 38 and 39) that show this is not the case. These examples provide a counterpoint to the work of [DDD $\left.{ }^{+} 19\right]$, which shows that the $\widetilde{H}_{i-1}\left(\mathcal{O}\left(P_{>j}\right) ; k\right)$ do detect the depth of $k[\Delta]$, and, in particular, can determine whether $\Delta$ is Cohen-Macaulay.

In general, equality need not hold in Theorem 2 ; depth $\widetilde{\Delta}_{i}$ can be any value between $\operatorname{dim} \widetilde{\Delta}_{i}$ and $\operatorname{depth} k[\Delta]-1$. However, we prove that one can often find a rank so that equality is achieved.

Proposition C. Let $(\Delta, \pi)$ be a balanced simplicial complex of dimension $d-1$, with ordered partition $\pi=\left(V_{1}, \ldots, V_{d}\right)$. If $\widetilde{H}_{\text {depth } k[\Delta]-1}(\Delta)=0$, then there is an $i \in[d]$ such that depth $k\left[\widetilde{\Delta}_{i}\right]=\operatorname{depth} k[\Delta]-1$.

Using Proposition $\mathrm{C}$, we provide a formula for depth $k[\Delta]$ for any balanced simplicial complex $\Delta$ (see Theorem 26).

Finally, the aformentioned results suggest a more concrete relationship between the reduced homologies of links in $\Delta$ and the $\widetilde{H}_{i-1}\left(\mathcal{O}\left(P_{>j}\right) ; k\right)$. For this relationship we provide the following formula for sums of reduced Euler characteristics of links. Our formula is analogous to those of [HN02, Section 2 Lemma 1 (i)] and [Swa05, Proposition 2.3].

Theorem D. Suppose $\Delta$ is pure and let $P$ be the face poset of $\Delta$. Write $\chi$ for Euler characteristic and $\tilde{\chi}$ for reduced Euler characteristic. Then

$$
\sum_{\substack{T \in \Delta \\|T|=j}} \tilde{\chi}\left(\mathrm{lk}_{\Delta}(T)\right)=\chi\left(\mathcal{O}\left(P_{>j}\right)\right)-\chi\left(\mathcal{O}\left(P_{>j-1}\right)\right) .
$$

We now describe the structure of our paper. In Section 2, we set notation and provide the algebraic and combinatorial background we appeal to throughout the paper. In Section 3, we prove Theorems A and B (see Theorems 16, 17 and 18). Section 4 contains a proof of Proposition C (Proposition 24) as well as a formula for depth $k[\Delta]$ (Theorem 26). In Section 5, we prove Theorem D (Theorem 30) and provide an application to Gorenstein* complexes. The last section discusses open problems related to this work and provides examples indicating the sharpness of our results.

\section{Background and Notation}

In this section we set notation and provide necessary background. Once and for all, fix the base field $k$. We let $\widetilde{H}_{i}$ denote $i$ th simplicial or singular homology, whichever is appropriate, always taken with respect to the field $k$. We use $\chi$ for Euler characteristic and $\tilde{\chi}$ for reduced Euler characteristic.

Given a simplicial complex $\Delta$, we write $k[\Delta]$ for its Stanley-Reisner ring over $k$. We write $V(\Delta)$ for the vertex set of $\Delta$, but, if $\Delta$ is clear from context, we generally write $V$ for $V(\Delta)$ and $n$ for $|V|$; we set $A:=k\left[x_{1}, \ldots, x_{n}\right]$. We write $f_{i}(\Delta)$ for the number of 
$i$-dimensional faces of $\Delta$, and $h_{i}(\Delta)$ for the $i$ th entry of the $h$-vector of $\Delta$; so $h_{i}(\Delta)=$ $\sum_{j=0}^{i}(-1)^{i-j}\left(\begin{array}{c}d-j \\ i-j\end{array}\right) f_{j-1}(\Delta)$. We let $\|\Delta\|$ denote the geometric realization of $\Delta$. We call $\Delta^{(k)}:=\{\sigma \in \Delta: \operatorname{dim} \sigma \leqslant k\}$ the $k$-skeleton of $\Delta$.

Given a subset $T \subseteq V(\Delta)$, we use $\left.\Delta\right|_{T}:=\{\sigma \in \Delta \mid \sigma \subseteq T\}$ for the induced subcomplex of $\Delta$ on $T$. We may then define the star, the anti-star, and the link of $T$, respectively, as follows:

$$
\begin{aligned}
\operatorname{st}_{\Delta} T & :=\{G \in \Delta \mid T \cup G \in \Delta\} \\
\operatorname{ast}_{\Delta} T & :=\{G \in \Delta \mid T \cap G=\varnothing\}=\left.\Delta\right|_{V-T} \\
\operatorname{lk}_{\Delta} T & :=\{G \in \Delta \mid T \cup G \in \Delta \text { and } T \cap G=\varnothing\}=\text { st }_{\Delta} T \cap \operatorname{ast}_{\Delta} T
\end{aligned}
$$

We note that $\mathrm{st}_{\Delta} T$ and $\mathrm{lk}_{\Delta} T$ are the void complex $\varnothing$ exactly when $T \notin \Delta$, and $\operatorname{lk}_{\Delta}(T)$ is the irrelevant complex $\{\varnothing\}$ exactly when $T$ is a facet of $\Delta$. On the other hand, $\operatorname{ast}_{\Delta}(T)$ is nonempty as long as long as $T \neq V$. Of import, $\mathrm{st}_{\Delta}(T)$ is a cone over $\mathrm{lk}_{\Delta}(T)$ for any $T \in \Delta$, and in particular is acyclic. When $T=\{v\}$, we abuse notation and write $\operatorname{st}_{\Delta}(v)$, $\operatorname{ast}_{\Delta}(v)$, and $\operatorname{lk}_{\Delta}(v)$.

Definition 3. We say that $J \subseteq V(\Delta)$ is an independent set for $\Delta$ if $\{a, b\} \notin \Delta$ for any $a, b \in J$ with $a \neq b$. Motivated by [Hib91], we say that $J \subseteq V(\Delta)$ is an excellent set for $\Delta$ if $J$ is an independent set for $\Delta$ and $J \cap F \neq \varnothing$ for every facet $F \in \Delta$. When $\Delta$ is clear from context, we simply say that $J$ is an independent set or that $J$ is an excellent set, as appropriate.

The main computational tools of this paper are two exact sequences recorded in the following propositions:

Proposition 4 ([DDD ${ }^{+} 19$, Lemma 4.2]). Suppose $b$ is a non-isolated vertex of $\Delta$. Then there is a Mayer-Vietoris exact sequence of the form

$$
\cdots \rightarrow \widetilde{H}_{i}(\Delta) \rightarrow \widetilde{H}_{i-1}\left(\operatorname{lk}_{\Delta}(b)\right) \rightarrow \widetilde{H}_{i-1}\left(\operatorname{ast}_{\Delta}(b)\right) \rightarrow \widetilde{H}_{i-1}(\Delta) \rightarrow \cdots .
$$

Proposition 5 ([DDD ${ }^{+} 19$, Proof of Lemma 4.3]). Suppose $\{x\} \subsetneq J \subsetneq V$ and $J$ is an independent set. Set $J^{\prime}=J-\{x\}$. Then there is a Mayer-Vietoris exact sequence of the form

$$
\cdots \rightarrow \widetilde{H}_{i}(\Delta) \rightarrow \widetilde{H}_{i-1}\left(\operatorname{ast}_{\Delta}(J)\right) \rightarrow \widetilde{H}_{i-1}\left(\operatorname{ast}_{\Delta}\left(J^{\prime}\right)\right) \oplus \widetilde{H}_{i-1}\left(\operatorname{ast}_{\Delta}(x)\right) \rightarrow \widetilde{H}_{i-1}(\Delta) \rightarrow \cdots
$$

We also consider algebraic properties of $k[\Delta]$; one can see [BH93] as a reference for this subject. We use $\operatorname{dim} k[\Delta]$ for the Krull dimension of the $\operatorname{ring} k[\Delta]$; so $\operatorname{dim} \Delta=$ $\operatorname{dim} k[\Delta]-1$. We write $d$ for $\operatorname{dim} k[\Delta]$ when $\Delta$ is clear from context. By $\operatorname{depth} k[\Delta]$ we mean the depth of the $k$-algebra $k[\Delta]$; for a combinatorial characterization of depth, see Proposition 7 . We say $\Delta$ is Cohen-Macaulay whenever $k[\Delta]$ is Cohen-Macaulay, that is, if $\operatorname{depth} k[\Delta]=\operatorname{dim} k[\Delta]$. Recall the following: 
Definition 6. A commutative Noetherian ring $R$ satisfies Serre's Condition, $\left(S_{\ell}\right)$, if, for all $\mathfrak{p} \in \operatorname{Spec} R$, depth $R_{\mathfrak{p}} \geqslant \min \left\{\ell, \operatorname{dim} R_{\mathfrak{p}}\right\}$.

We say $\Delta$ satisfies $\left(S_{\ell}\right)$ if $k[\Delta]$ does. Every simplicial complex satisfies $\left(S_{1}\right)$, and a simplicial complex satisfies $\left(S_{d}\right)$ if and only if it is Cohen-Macaulay.

The following is an immediate consequence of Hochster's formula ([BH93, Theorem 5.3.8]) and gives a useful characterization of depth for Stanley-Reisner rings in terms of reduced homologies of links:

Proposition 7. Let $\Delta$ be a simplicial complex. Then depth $k[\Delta] \geqslant t$ if and only if $\widetilde{H}_{i-1}\left(\mathrm{lk}_{\Delta}(T)\right)=0$ for all $T \in \Delta$ with $i+|T|<t$.

The corresponding result for $\left(S_{\ell}\right)$ can be found in [Ter07]:

Proposition 8 ([Ter07]). Let $\Delta$ be a simplicial complex of dimension $d-1$. Then $\Delta$ satisfies $\left(S_{\ell}\right)$ for $\ell \geqslant 2$ if and only if $\widetilde{H}_{i-1}\left(\operatorname{lk}_{\Delta}(T)\right)=0$ whenever $i+|T|<d$ and $0 \leqslant i<\ell$. In particular, $\left(S_{\ell}\right)$ complexes are pure if $\ell \geqslant 2$.

One can obtain similar characterizations for other algebraic properties of $k[\Delta]$. Define core $V(\Delta):=\left\{v \in V(\Delta) \mid \operatorname{st}_{\Delta}(v) \neq \Delta\right\}$ and set core $\Delta:=\left.\Delta\right|_{\operatorname{core} V(\Delta)}$. We say that $\Delta$ is Gorenstein if the ring $k[\Delta]$ is Gorenstein; if, in addition, core $\Delta=\Delta$, we say that $\Delta$ is Gorenstein*. One has the following, see [BH93, Theorem 5.6.1]:

Theorem 9. A simplicial complex $\Delta$ is Gorenstein* if and only if

$$
\widetilde{H}_{i-1}\left(\mathrm{lk}_{\Delta}(T)\right) \cong \begin{cases}k & \text { if } i=d-|T| \\ 0 & \text { if } i \neq d-|T|\end{cases}
$$

Now, let $P$ be a finite poset. If $p \in P$, we let ht $(p)$ denote the length of a longest chain $p_{1} \prec p_{2} \prec \cdots \prec p_{i}=p$ and let ht $P:=\max \{$ ht $p \mid p \in P\}$. We denote by $P_{>j}$ the poset obtained by restricting to elements $p \in P$ so that ht $p>j$. The order complex of $P$, denoted $\mathcal{O}(P)$, is the simplicial complex on $P$ consisting of all chains of elements in $P$. Let $\mathcal{F}(\Delta)$ denote the face poset of $\Delta$. We set $[\Delta]_{>j}:=\mathcal{O}\left(\mathcal{F}(\Delta)_{>j}\right)$. We note that when $j=0,[\Delta]_{>0}$ is the barycentric subdivision of $\Delta$. The following is well known (see [Gib10, Corollary 5.7], for example):

Lemma 10. The realization $\|\Delta\|$ is homeomorphic to $\left\|[\Delta]_{>0}\right\|$. In particular, $\widetilde{H}_{i}(\Delta) \cong$ $\widetilde{H}_{i}\left([\Delta]_{>0}\right)$ for all $i$.

We let $\rho: \Delta-\{\varnothing\} \rightarrow V\left([\Delta]_{>0}\right)$ be the map which sends $T$ to itself viewed as a vertex of $[\Delta]_{>0}$.

There are several advantages of working with $[\Delta]_{>k}$. For instance, we have the following result of $\left[\mathrm{DDD}^{+} 19\right]$ :

Lemma 11. Let $T \in \Delta$. Then $\left[\mathrm{lk}_{\Delta}(T)\right]_{>0} \cong \mathrm{lk}_{[\Delta]_{>|T|-1}}(\rho(T))$ as simplicial complexes. In particular, $\widetilde{H}_{i}\left(\mathrm{lk}_{\Delta}(T)\right) \cong \widetilde{H}_{i}\left(\mathrm{lk}_{[\Delta]_{>|T|-1}}(\rho(T))\right)$ for each $i$. 
Definition 12. A balanced simplicial complex is a pair $(\Delta, \pi)$ satisfying:

1. $\Delta$ is $d-1$ dimensional simplicial complex on a vertex set $V$.

2. $\pi=\left(V_{1}, \ldots, V_{d}\right)$ is an ordered partition of $V$.

3. For every facet $F \in \Delta$ and every $i \in[d],\left|F \cap V_{i}\right| \leqslant 1$.

Balanced simplicial complexes were introduced by Stanley in [Sta79]. One can find more information on balanced simplicial complexes in [BFS87, BGS82, Gar80]; [Sta96] gives a more modern treatment of the subject. An important property of balanced simplicial complexes is that each $V_{i}$ is an independent set for $\Delta$, and, if $\Delta$ is pure, the $V_{i}$ are excellent sets for $\Delta$. If $(\Delta, \pi)$ is a balanced simplicial complex with $\pi=\left(V_{1}, \ldots, V_{d}\right)$, and if $S \subseteq[d]$, we define the $S$-rank selected subcomplex of $\Delta$ to be the complex $\Delta_{S}:=\left.\Delta\right|_{\bigcup_{i \in S} V_{i}}$; for notational convenience, we also set $\widetilde{\Delta}_{S}=\Delta_{[d]-S}$. If $(\Delta, \pi)$ is a balanced simplicial complex, we often suppress the ordered partition $\pi$ and simply refer to $\Delta$ as a balanced simplicial complex; in this case we always write $\pi=\left(V_{1}, \ldots, V_{d}\right)$ for the corresponding ordered partition.

Now, let $P$ be a finite poset. If we set $V_{i}:=\{p \mid \operatorname{ht}(p)=i\}$ and $\pi=\left(V_{1}, \ldots, V_{\text {ht } P}\right)$, then $(\mathcal{O}(P), \pi)$ is a balanced simplicial complex. In particular, this means $[\Delta]_{>j}$ is always a balanced simplicial complex for any $j$.

Finally, we recall the higher nerve complexes of $\left[\mathrm{DDD}^{+} 19\right]$ :

Definition 13. Let $\left\{A_{1}, A_{2}, \ldots, A_{r}\right\}$ be the collection of facets of $\Delta$. The simplicial complex

$$
N_{i}(\Delta):=\left\{F \subseteq[r]:\left|\bigcap_{j \in F} A_{j}\right| \geqslant i\right\}
$$

is called the ith Nerve Complex of $\Delta$. We refer to the $N_{i}(\Delta)$ as the higher Nerve Complexes of $\Delta$. We note that $N_{0}(\Delta)=2^{[r]}$ and $N_{1}(\Delta)$ is the classical nerve complex of $\Delta$.

Higher nerve complexes capture important homological information about $\Delta$. For our purposes, the important properties of higher nerve complexes can be summarized as follows:

Theorem 14 ([DDD ${ }^{+} 19$, Theorems 1.3 and 2.8]). Let $\Delta$ be a simplicial complex of dimension $d-1$. Then

1) $\widetilde{H}_{i-1}\left(N_{j+1}(\Delta)\right)=0$ for $i+j>d$ and $1 \leqslant j \leqslant d$.

2) $\operatorname{depth} k[\Delta]=\inf \left\{i+j: \widetilde{H}_{i-1}\left(N_{j+1}(\Delta)\right)=0\right\}$.

3) For $i \geqslant 0$,

$$
f_{i}(\Delta)=\sum_{j=i}^{d-1}\left(\begin{array}{l}
j \\
i
\end{array}\right) \chi\left(N_{j+1}(\Delta)\right) .
$$

4) $\widetilde{H}_{i}\left([\Delta]_{>k}\right) \cong \widetilde{H}_{i}\left(N_{k+1}(\Delta)\right)$ for any $i$ and any $k$. 


\section{Rank Selection Theorems for Serre's Condition}

In this section we prove some general statements and use them to derive Theorems A and B. Throughout this section we let $\Delta$ be a simplicial complex of dimension $d-1$ on the vertex set $V$.

Lemma 15. Suppose $J \subseteq V$ is excellent and that $\Delta$ satisfies $\left(S_{\ell}\right)$. Set $\widetilde{\Delta}:=\operatorname{ast}_{\Delta}(J)$. Then $\widetilde{\Delta}$ satisfies $\left(S_{\ell}\right)$.

Proof. We proceed by induction on $\ell$. The claim is clear when $\ell=1$, since every simplicial complex satisfies $\left(S_{1}\right)$. So, suppose we know the result for all $1 \leqslant j \leqslant \ell$ and suppose $\Delta$ satisfies $\left(S_{\ell+1}\right)$. The inductive hypothesis gives us that $\widetilde{\Delta}$ satisfies $\left(S_{\ell}\right)$, and we will show $\widetilde{\Delta}$ satisfies $S_{\ell+1}$; the Lemma will then follow from induction.

By Proposition 8, we have that $\widetilde{H}_{i-1}\left(\operatorname{lk}_{\Delta}(T)\right)=0$ whenever $i+|T|<d$ and $0 \leqslant i \leqslant \ell$, and that $\widetilde{H}_{i-1}\left(\operatorname{lk}_{\widetilde{\Delta}}(T)\right)=0$ whenever $i+|T|<d-1$ and $0 \leqslant i<\ell$. We need only show that $\widetilde{H}_{\ell-1}\left(\operatorname{lk}_{\widetilde{\Delta}}(T)\right)=0$ for all $T \in \widetilde{\Delta}$ with $\ell+|T|<d-1$.

Pick $T \in \widetilde{\Delta}$ such that $\ell+|T|<d-1$. Let $\sigma \supseteq T$ be a facet of $\Delta$. Since $J$ is excellent, there is a $b \in J \cap \sigma$, and thus $\{b\} \cup T \in \Delta$. Since $b \notin T$, this means $b \in \operatorname{lk}_{\Delta}(T)$. Note that $T \cup\{b\}$ cannot be a facet of $\Delta$, since this would mean $|T|+1=d$, whilst $\ell+|T|<d-1$. Set $S=J \cap V\left(\mathrm{lk}_{\Delta}(T)\right)$; then we have $\operatorname{lk}_{\widetilde{\Delta}}(T)=\operatorname{ast}_{\mathrm{lk}_{\Delta}(T)}(S)$. By Proposition 4, we have, for any $b \in S$, the exact sequence:

$$
\widetilde{H}_{\ell}\left(\operatorname{ast}_{\mathrm{lk}_{\Delta}(T)}(b)\right) \stackrel{i_{b}^{*}}{\rightarrow} \widetilde{H}_{\ell}\left(\mathrm{lk}_{\Delta}(T)\right) \rightarrow \widetilde{H}_{\ell-1}\left(\operatorname{lk}_{\mathrm{lk}_{\Delta}(T)}(b)\right) \rightarrow \widetilde{H}_{\ell-1}\left(\operatorname{ast}_{\mathrm{lk}_{\Delta}(T)}(b)\right) \rightarrow \widetilde{H}_{\ell-1}\left(\mathrm{lk}_{\Delta}(T)\right)
$$

where $i_{b}^{*}$ is the induced map coming from the inclusion $i_{b}: \operatorname{ast}_{\mathrm{lk}_{\Delta}(T)}(b) \hookrightarrow \mathrm{lk}_{\Delta}(T)$. Since $\operatorname{lk}_{\mathrm{lk}_{\Delta}(T)}(b)=\mathrm{lk}_{\Delta}(T \cup\{b\})$ and since $\ell+|T|<d-1$, we have $\widetilde{H}_{\ell-1}\left(\operatorname{lk}_{\mathrm{lk}_{\Delta}(T)}(b)\right)=0$. Since $\widetilde{H}_{\ell-1}\left(\operatorname{lk}_{\Delta}(T)\right)=0$, we obtain $\widetilde{H}_{\ell-1}\left(\operatorname{ast}_{\mathrm{lk}_{\Delta}(T)}(b)\right)=0$ and that $i_{b}^{*}$ is surjective, from exactness.

Now, since $J$ is an independent set in $\Delta, S$ is an independent set in $\operatorname{lk}_{\Delta}(T)$. We claim that $\widetilde{H}_{\ell-1}\left(\operatorname{ast}_{\mathrm{k}_{\Delta}(T)}(I)\right)=0$ for any $\varnothing \subsetneq I \subseteq S$. To see this, we induct on $|I|$. Note that the claim is true when $|I|=1$, from above. Now suppose the claim is true for every $I$ with $|I|=k$, and suppose we are given an $I$ with $|I|=k+1$. Write $I=L \cup\{a\}$ so that $|L|=k$. By Proposition 5 we have the exact sequence

$$
\begin{aligned}
& \widetilde{H}_{\ell}\left(\operatorname{ast}_{\mathrm{k}_{\Delta}(T)}(a)\right) \oplus \widetilde{H}_{\ell}\left(\operatorname{ast}_{\mathrm{k}_{\Delta}(T)}(L)\right) \longrightarrow \widetilde{H}_{\ell}^{*}-k^{*}\left.\longrightarrow \widetilde{\operatorname{lk}}_{\Delta}(T)\right) \longrightarrow \\
&\left.\longrightarrow \widetilde{H}_{\ell-1}\left(\operatorname{ast}_{\mathrm{lk}_{\Delta}(T)}(I)\right) \longrightarrow \operatorname{ast}_{\mathrm{lk}_{\Delta}(T)}(a)\right) \oplus \widetilde{H}_{\ell-1}\left(\operatorname{ast}_{\mathrm{lk}_{\Delta}(T)}(L)\right)
\end{aligned}
$$

where $k^{*}$ is the induced map coming from the inclusion $k: \operatorname{ast}_{\mathrm{lk}_{\Delta}(T)}(L) \hookrightarrow \operatorname{lk}_{\Delta}(T)$.

By the inductive hypothesis, we have that $\widetilde{H}_{\ell-1}\left(\operatorname{ast}_{\mathrm{lk}_{\Delta}(T)}(a)\right) \oplus \widetilde{H}_{\ell-1}\left(\operatorname{ast}_{\mathrm{lk}_{\Delta}(T)}(L)\right)=$ 0 . As we saw previously, $i_{a}^{*}$ is surjective so that $i_{a}^{*}-k^{*}$ is as well. Thus we obtain $\widetilde{H}_{\ell-1}\left(\operatorname{ast}_{\mathrm{kk}_{\Delta}(T)}(I)\right)=0$ from exactness. Induction then gives us that $\widetilde{H}_{\ell-1}\left(\operatorname{ast}_{\mathrm{lk}_{\Delta}(T)}(S)\right)=$ $\widetilde{H}_{\ell-1}\left(\operatorname{lk}_{\widetilde{\Delta}}(T)\right)=0$, and thus $\widetilde{\Delta}$ satisfies $\left(S_{\ell+1}\right)$. 
Theorems A and B (1) now follow as quick consequences of Lemma 15:

Theorem 16. Let $\Delta$ be a balanced simplicial complex. If $\Delta$ satisfies $\left(S_{\ell}\right)$, then $\Delta_{S}$ satisfies $\left(S_{\ell}\right)$ for any $S \subseteq[d]$.

Proof. There is nothing to prove when $\ell=1$. When $\ell \geqslant 2, \Delta$ is pure, and the result follows by applying Lemma 15 inductively on each $i \in[d]-S$.

Theorem 17. If $P$ is a finite poset satisfying $\left(S_{\ell}\right)$, then $\widetilde{H}_{i-1}\left(\mathcal{O}\left(P_{>j}\right)\right)=0$ whenever $i+j<d$ and $0 \leqslant i<\ell$. In particular, if $\Delta$ is a simplicial complex satisfying $\left(S_{\ell}\right)$, then $\widetilde{H}_{i-1}\left([\Delta]_{>j}\right)=0$ whenever $i+j<d$ and $0 \leqslant i<\ell$.

Proof. Suppose $P$ is $\left(S_{\ell}\right)$. By Theorem $16, \mathcal{O}\left(P_{>j}\right)$ satisfies $\left(S_{\ell}\right)$ for each $0 \leqslant j \leqslant d-1$. In particular, $\widetilde{H}_{i-1}\left(\mathcal{O}\left(P_{>j}\right)\right)=0$ for $i<d-j$ and $0 \leqslant i<\ell$. It only remains to remark that if $\Delta$ is a simplicial complex satisfying $\left(S_{\ell}\right)$, then, since $\|\Delta\| \cong\left\|[\Delta]_{>0}\right\|$ and since $\left(S_{\ell}\right)$ is a topological property ([Yan11, Theorem $\left.\left.4.4(\mathrm{~d})\right]\right),[\Delta]_{>0}$ satisfies $\left(S_{\ell}\right)$.

Remarkably, Theorem 17 admits a partial converse (Theorem B (2)) when $P$ is the face poset of a simplicial complex.

Theorem 18. If $\widetilde{H}_{i-1}\left([\Delta]_{>j}\right)=0$ whenever $i+j<d$ and $0 \leqslant i \leqslant \ell$, then $\Delta$ satisfies $\left(S_{\ell}\right)$.

Proof. We induct on $\ell$, using a similar approach to that of Lemma 15. There is nothing to prove when $\ell=1$ and we may assume $\ell \leqslant d$. Suppose we know the result for $\ell$ and suppose $\widetilde{H}_{i-1}\left([\Delta]_{>j}\right)=0$ whenever $i+j<d$ and $0 \leqslant i \leqslant \ell+1$. From induction hypothesis, we have that $\Delta$ satisfies $\left(S_{\ell}\right)$. Note that we assumed, in particular, that $\widetilde{H}_{0}\left([\Delta]_{>j}\right)=0$ whenever $j<d-1$. Thus, no facet of $\Delta$ can have cardinality less than or equal to $d-1$, i.e., $\Delta$ is pure. Since $\Delta$ is $\left(S_{\ell}\right)$, we have $\widetilde{H}_{i-1}\left(\operatorname{lk}_{\Delta}(T)\right)=0$ whenever $i+|T|<d$ and $0 \leqslant i<\ell$, and we need only show that $\widetilde{H}_{\ell-1}\left(\operatorname{lk}_{\Delta}(T)\right)=0$ whenever $|T|<d-\ell$. To see this, we proceed by induction on $|T|$. When $|T|=0$, we have $\widetilde{H}_{\ell-1}(\operatorname{lk}(T))=\widetilde{H}_{\ell-1}(\Delta)=\widetilde{H}_{\ell-1}\left([\Delta]_{>0}\right)=0$. Suppose $\widetilde{H}_{\ell-1}(\operatorname{lk}(T))=0$ whenever $j=|T|<d-\ell$, and consider $T \in \Delta$ with $j+1=|T|<d-\ell$.

Letting $S=\{\rho(T)|T \in \Delta| T \mid,=j+1\}$ and writing $S=I \cup\{\rho(T)\}$, we have, by Proposition 5, the exact sequence

$$
\widetilde{H}_{\ell-1}\left([\Delta]_{>j+1}\right) \longrightarrow \widetilde{H}_{\ell-1}\left(\operatorname{ast}_{[\Delta]_{>j}}(\rho(T))\right) \oplus \widetilde{H}_{\ell-1}\left(\operatorname{ast}_{[\Delta]_{>j}}(I)\right) \longrightarrow \widetilde{H}_{\ell-1}\left([\Delta]_{>j}\right)
$$

Since $\widetilde{H}_{\ell-1}\left([\Delta]_{>j+1}\right)=0=\widetilde{H}_{\ell-1}\left([\Delta]_{>j}\right)$, it follows from the exact sequence above that $\widetilde{H}_{\ell-1}\left(\operatorname{ast}_{[\Delta]_{>j}}(\rho(T))\right) \oplus \widetilde{H}_{\ell-1}\left(\operatorname{ast}_{[\Delta]_{>j}}(I)\right)=0$. In particular, $\widetilde{H}_{\ell-1}\left(\operatorname{ast}_{[\Delta]_{>j}}(\rho(T))\right)=0$.

As $\Delta$ is pure, $T$ is not a facet, and so $\rho(T)$ is a non-isolated vertex of $[\Delta]_{>j}$. By Proposition 4, we have the exact sequence

$$
\widetilde{H}_{\ell}\left([\Delta]_{>j}\right) \rightarrow \widetilde{H}_{\ell-1}\left(\operatorname{lk}_{[\Delta]_{>j}}(\rho(T))\right) \rightarrow \widetilde{H}_{\ell-1}\left(\operatorname{ast}_{[\Delta]_{>j}}(\rho(T))\right) \rightarrow \widetilde{H}_{\ell-1}\left([\Delta]_{>j}\right)
$$


Since $\widetilde{H}_{\ell-1}\left(\operatorname{ast}_{[\Delta]_{>j}}(\rho(T))\right)=0=\widetilde{H}_{\ell}\left([\Delta]_{>j}\right)$, we have $\widetilde{H}_{\ell-1}\left(\operatorname{lk}_{[\Delta]_{>j}}(\rho(T))\right)=0=$ $\widetilde{H}_{\ell-1}(\operatorname{lk}(T))$, by Proposition 7 . Thus, $\Delta$ satisfies $\left(S_{\ell+1}\right)$, and the result follows from induction.

Remark 19. When $\ell=2$, the conclusion of Theorem 17 is equivalent to $\widetilde{H}_{0}\left([\Delta]_{>d-2}\right)=0$, since for a pure complex connectivity of $[\Delta]_{>j}$ implies connectivity of $[\Delta]_{>j-1}$.

Remark 20. Since, by Theorem $14(4), \widetilde{H}_{i-1}\left([\Delta]_{>j}\right) \cong \widetilde{H}_{i-1}\left(N_{j+1}(\Delta)\right)$ for any $i$ and $j$, Theorems 17 and 18 also serve as a version of Theorem $14(2)$ for $\left(S_{\ell}\right)$, and in fact recover the Cohen-Macaulay case of Theorem 14 (2).

Remark 21. Theorems 17 and 18 show that the reduced homologies of the $[\Delta]_{>j}$ pin the largest $\ell$ for which $\Delta$ satisfies $\left(S_{\ell}\right)$ to two possible consecutive values. As Examples 38 and 39 show, the reduced homologies of the $[\Delta]_{>j}$ alone cannot determine which of these values $\ell$ actually is. We would be quite interested to know what information can be used in tandem with the $\widetilde{H}_{i-1}\left([\Delta]_{>j}\right)$ to determine this value; see Question 41.

We conclude this section with an example illustrating the application of the results from this section.

Example 22. Consider the complex $\Delta$ with facets:

$$
\begin{gathered}
\{3,4,5,6\},\{4,5,6,7\},\{3,5,6,7\},\{2,5,6,7\},\{1,3,6,7\},\{2,4,5,7\}, \\
\{2,3,4,7\},\{2,3,6,7\},\{1,3,4,7\},\{1,3,4,6\},\{2,3,4,5\}
\end{gathered}
$$

We organize the dimensions of the $\widetilde{H}_{i-1}\left([\Delta]_{>j}\right)$ into the following table:

\begin{tabular}{|l|c|c|c|c|}
\hline & $\widetilde{H}_{0}$ & $\widetilde{H}_{1}$ & $\widetilde{H}_{2}$ & $\widetilde{H}_{3}$ \\
\hline$[\Delta]_{>0}$ & 0 & 0 & 1 & 0 \\
{$[\Delta]_{>1}$} & 0 & 0 & 1 & 0 \\
{$[\Delta]_{>2}$} & 0 & 9 & 0 & 0 \\
{$[\Delta]_{>3}$} & 10 & 0 & 0 & 0 \\
\hline
\end{tabular}

Theorem 18 implies that $\Delta$ satisfies $\left(S_{2}\right)$, and Theorem 17 implies that $\Delta$ does not satisfy $\left(S_{4}\right)$, i.e., that $\Delta$ is not Cohen-Macaulay. However, neither of these Theorems can determine whether $\Delta$ satisfies $\left(S_{3}\right)$; one can check that this $\Delta$ does not satisfy $\left(S_{3}\right)$. In fact, $\mathrm{lk}_{\Delta}(T)$ is acyclic when $|T|=1$ except when $T=\{3\}$ or $T=\{4\}$ which each have exactly one nonzero reduced homology: $\widetilde{H}_{1}\left(\mathrm{lk}_{\Delta}(\{4\})\right) \cong \widetilde{H}_{2}\left(\mathrm{lk}_{\Delta}(\{3\}) \cong k\right.$. Comparing these to the table above suggests a more general relationship between reduced homologies of links and the $\widetilde{H}_{i-1}\left([\Delta]_{>j}\right)$. This relationship is the main subject of the next section. In fact, one can find examples having a table, as above, that is identical to that of $\Delta$, but which do satisfy $\left(S_{3}\right)$ (See Examples 38 and 39 for a version of this behavior in smaller dimension). For instance, one can generate such an example by replacing the facet $\{2,3,4,5\}$ of $\Delta$ with $\{2,3,4,6\}$. What makes this behavior possible is that the $\widetilde{H}_{i-1}\left([\Delta]_{>j}\right)$ only relate to homologies of links in general through Euler characteristic (see Theorem 30), so there is a loss of information in this relationship. In particular, the $\widetilde{H}_{i-1}\left([\Delta]_{>j}\right)$ cannot detect whether the vertex links are all acyclic, or whether, as with this example, link homologies are in appropriate degrees to sum to zero in the formula of Theorem 30. 


\section{Depth of Rank Selected Subcomplexes}

In this section, we explore the behavior of depth when passing to rank selected subcomplexes. In particular, we prove Proposition $\mathrm{C}$ and use it to provide a formula for the depth of any balanced simplicial complex in Theorem 26. Throughout this section we let $\Delta$ be a simplicial complex of dimension $d-1$ on the vertex set $V$.

The following lemma follows from [Hib91, Proposition 2.8] and a slightly weaker version can be found in [Mun84, Theorem 6.4]:

Lemma 23. Suppose $J \subseteq V$ is an independent set. Set $\widetilde{\Delta}=\operatorname{ast}_{\Delta}(J)$. Then $\operatorname{depth} \widetilde{\Delta} \geqslant$ $\operatorname{depth} \Delta-1$.

We first provide a variation on this lemma:

Lemma 24. Let depth $\Delta=\ell$ and suppose $\widetilde{H}_{\ell-1}(\Delta)=0$. Choose $T \in \Delta$ of minimal cardinality such that $\widetilde{H}_{\ell-|T|-1}\left(\mathrm{lk}_{\Delta}(T)\right) \neq 0$ (that such a $T$ exists follows from Proposition 7). Let $J \subseteq V$ be an independent set and suppose $T=T^{\prime} \cup\{b\}$ with $b \in J$. Set $\widetilde{\Delta}=\operatorname{ast}_{\Delta}(J)$. Then $\widetilde{H}_{\ell-\left|T^{\prime}\right|-2}\left(\mathrm{lk}_{\widetilde{\Delta}}\left(T^{\prime}\right)\right) \neq 0$. In particular, depth $\widetilde{\Delta}=\ell-1$.

Proof. If $T$ is a facet of $\Delta$, then we have that $|T|=\ell$ by minimality, and, as $\operatorname{lk}_{\Delta}(T)=$ $\mathrm{lk}_{\mathrm{lk}_{\Delta}\left(T^{\prime}\right)}(b)$, that $\{b\}$ is a facet of $\mathrm{lk}_{\Delta}\left(T^{\prime}\right)$. By our minimality hypothesis, $\widetilde{H}_{0}\left(\mathrm{lk}_{\Delta}\left(T^{\prime}\right)\right)=0$. It follows that $\mathrm{lk}_{\Delta}\left(T^{\prime}\right)$ is a simplex with facet $\{b\}$, and so $\operatorname{lk}_{\widetilde{\Delta}}\left(T^{\prime}\right)=\operatorname{ast}_{\mathrm{k}_{\Delta}\left(T^{\prime}\right)}(b)=\{\varnothing\}$. Thus $T^{\prime}$ is a facet of $\widetilde{\Delta}$, and so $\widetilde{H}_{\ell-1-\left|T^{\prime}\right|-1}\left(\mathrm{l}_{\widetilde{\Delta}}\left(T^{\prime}\right)\right)=\widetilde{H}_{-1}\left(\mathrm{lk}_{\widetilde{\Delta}}\left(T^{\prime}\right)\right) \neq 0$.

Otherwise, if $T$ is not a facet of $\Delta$, set $S=J \cap V\left(\mathrm{lk}_{\Delta}\left(T^{\prime}\right)\right)$ and note that $\operatorname{lk}_{\widetilde{\Delta}}\left(T^{\prime}\right)=$ $\operatorname{ast}_{\mathrm{lk}_{\Delta}\left(T^{\prime}\right)}(S)$. Lemma 4 gives the following exact sequence

$$
\widetilde{H}_{\ell-|T|}\left(\operatorname{lk}_{\Delta}\left(T^{\prime}\right)\right) \rightarrow \widetilde{H}_{\ell-|T|-1}\left(\operatorname{lk}_{\mathrm{lk}_{\Delta}\left(T^{\prime}\right)}(b)\right) \rightarrow \widetilde{H}_{\ell-|T|-1}\left(\operatorname{ast}_{\mathrm{lk}_{\Delta}\left(T^{\prime}\right)}(b)\right) \rightarrow \widetilde{H}_{\ell-|T|-1}\left(\operatorname{lk}_{\Delta}\left(T^{\prime}\right)\right)
$$

By minimality of $|T|$ and Proposition 7 , we have $\widetilde{H}_{\ell-|T|}\left(\mathrm{lk}_{\Delta}\left(T^{\prime}\right)\right)=\widetilde{H}_{\ell-|T|-1}\left(\mathrm{lk}_{\Delta}\left(T^{\prime}\right)\right)=$ 0. Thus, $\widetilde{H}_{\ell-|T|-1}\left(\operatorname{lk}_{\mathrm{lk}_{\Delta}\left(T^{\prime}\right)}(b)\right) \cong \widetilde{H}_{\ell-|T|-1}\left(\operatorname{ast}_{\mathrm{k}_{\Delta}\left(T^{\prime}\right)}(b)\right)$. But, $\operatorname{lk}_{\mathrm{lk}_{\Delta}\left(T^{\prime}\right)}(b)=\operatorname{lk}_{\Delta}\left(T^{\prime} \cup\{b\}\right)=$ $\mathrm{lk}_{\Delta}(T)$, and so, in particular, $\widetilde{H}_{\ell-|T|-1}\left(\operatorname{ast}_{\mathrm{kk}_{\Delta}\left(T^{\prime}\right)}(b)\right) \neq 0$.

But now, $\left[\mathrm{DDD}^{+} 19\right.$, Lemma 4.3$]$ gives that

$$
\widetilde{H}_{i-|T|-1}\left(\operatorname{ast}_{\mathrm{lk}_{\Delta}\left(T^{\prime}\right)}(S)\right) \cong \bigoplus_{x \in S} \widetilde{H}_{i-|T|-1}\left(\operatorname{ast}_{\mathrm{lk}_{\Delta}\left(T^{\prime}\right)}(x)\right)
$$

and in particular that $\operatorname{lk}_{\widetilde{\Delta}}\left(T^{\prime}\right)$ is nonzero. That depth $\widetilde{\Delta}=\ell-1$ now follows from Lemma 23 and Proposition 7.

Proposition 25. Let $\Delta$ be a balanced simplicial complex with depth $\Delta=\ell$. Suppose $\widetilde{H}_{\ell-1}(\Delta)=0$. Then there exists an $i$ such that $\operatorname{depth}_{\operatorname{ast}_{\Delta}}\left(V_{i}\right)=\ell-1$.

Proof. This follows immediately from Lemma 24.

With these results in hand, we now provide a formula for depth $\Delta$. 
Theorem 26. Suppose $\Delta$ is a balanced simplicial complex with ordered partition $\pi=$ $\left(V_{1}, \ldots, V_{d}\right)$. Then

$$
\operatorname{depth} \Delta=\min \left\{i+|S| \mid \widetilde{H}_{i-1}\left(\widetilde{\Delta}_{S}\right) \neq 0\right\}
$$

where the minimum is taken over all $i$ and all $S \subseteq[d]$.

Proof. That

$$
\operatorname{depth} \Delta \leqslant \min \left\{i+|S| \mid \widetilde{H}_{i-1}\left(\widetilde{\Delta}_{S}\right) \neq 0\right\}
$$

follows at once from Lemma 23, so we need only concern ourselves with the reverse inequality. We proceed by induction on depth $\Delta$, noting that the claim is clear when $\operatorname{depth} \Delta=0$, that is, when $\Delta=\{\varnothing\}$. Suppose depth $\Delta=\ell$. The claim is clear if $\widetilde{H}_{\ell-1}(\Delta) \neq 0$, so we may suppose this is not the case. By Proposition 25 , there is an $i$ with $\operatorname{depth}_{\operatorname{ast}}\left(V_{i}\right)=\ell-1$. From the inductive hypothesis, we have $\ell-1=\min \{i+|S| \mid$ $\left.\widetilde{H}_{i-1}\left(\operatorname{ast}_{\Delta}\left(V_{i}\right)_{[d]-S}\right)\right\}$. In particular, there is an $S \subseteq[d-1]$ with $\widetilde{H}_{\ell-|S|-2}\left(\operatorname{ast}_{\Delta}\left(V_{i}\right)\right)=$ $\widetilde{H}_{\ell-|S \cup\{i\}|-1}\left(\widetilde{\Delta}_{|S \cup\{i\}|}\right) \neq 0$, and the result follows.

Corollary 27. Let $P$ be a finite poset. For any $S \subseteq\{1, \ldots$, ht $P\}$, let $\widetilde{P}_{S}$ denote the poset obtained by restricting $P$ to elements whose height is not in $S$. Then

$$
\operatorname{depth} \mathcal{O}(P)=\min \left\{i+|S| \mid \widetilde{H}_{i-1}\left(\mathcal{O}\left(\widetilde{P}_{S}\right)\right) \neq 0\right\} .
$$

In particular, for any simplicial complex $\Delta$, we can compute depth $k[\Delta]$ by taking $P$ to be the face poset of $\Delta$.

\section{$5 \quad$ Euler Characteristics of Links and Truncated Posets}

Motivated by the previous sections, this section focuses on exploring a more general relationship between the reduced homology groups $\widetilde{H}_{i-1}\left([\Delta]_{>j}\right)$ and reduced homologies of links in $\Delta$. This relationship in made concrete in Theorem $\mathrm{D}$, to which we now shift our attention. Throughout this section we continue to let $\Delta$ be a simplicial complex of dimension $d-1$ and we set $F_{k}=\{T \in \Delta,|T|=k\}$.

Lemma 28. Suppose $\Delta$ is pure. Then

$$
\sum_{T \in F_{k}} f_{i-1}\left(\mathrm{lk}_{\Delta}(T)\right)=\left(\begin{array}{c}
i+k \\
k
\end{array}\right) f_{i+k-1}(\Delta) .
$$

Proof. Note that $\left(\begin{array}{c}i+k \\ k\end{array}\right)$ is the number of $(k-1)$-dimensional faces contained in each $(i+$ $k-1)$-dimensional face. Thus the right hand side counts each $(i+k-1)$-dimensional face exactly once for each of its subfaces of dimension $k-1$. On the other hand, $f_{i-1}\left(\mathrm{lk}_{\Delta}(T)\right)$ counts each $(i+k-1)$-dimensional face containing $T$. Thus on the left hand side, each $(i+k-1)$-dimensional face is also counted exactly once for each $(k-1)$-dimensional face it contains, and so the sides are equal. 
As in [HN02, Section 2 Lemma 1 (i)] and [Swa05, Proposition 2.3], one can combine this with Theorem 1.3 (3) to obtain a formula for $\sum_{\substack{T \in \Delta \\|T|=k}} h_{i}\left(\operatorname{lk}_{\Delta}(T)\right)$ in terms of Euler characteristics of higher nerves. We follow a similar approach to obtain a particularly simple formula for $\sum_{\substack{T \in \Delta \\|T|=k}} \tilde{\chi}\left(\mathrm{lk}_{\Delta}(T)\right)$. To do this we need the following identity:

Lemma 29. For any positive integer $k$ and any nonnegative integer $j$, we have

$$
\sum_{i=0}^{j}(-1)^{i+1}\left(\begin{array}{c}
i+k \\
k
\end{array}\right)\left(\begin{array}{cl}
j \\
i+k-1
\end{array}\right)= \begin{cases}-1 & j=k-1 \\
1 & j=k \\
0 & j \neq k, k-1\end{cases}
$$

Proof. By Pascal's identity, we have that $\sum_{i=0}^{j}(-1)^{i+1}\left(\begin{array}{c}i+k \\ k\end{array}\right)\left(\begin{array}{c}j \\ i+k-1\end{array}\right)$ equals

$$
\sum_{i=0}^{j-k+1}(-1)^{i+1}\left(\begin{array}{c}
i+k-1 \\
k-1
\end{array}\right)\left(\begin{array}{c}
j \\
i+k-1
\end{array}\right)+\sum_{i=0}^{j-k+1}(-1)^{i+1}\left(\begin{array}{c}
i+k-1 \\
k
\end{array}\right)\left(\begin{array}{c}
j \\
i+k-1
\end{array}\right)
$$

Applying the subset of a subset identity to both terms, this equals

$$
\begin{aligned}
& \sum_{i=0}^{j-k+1}(-1)^{i+1}\left(\begin{array}{c}
j \\
k-1
\end{array}\right)\left(\begin{array}{c}
j-k+1 \\
i
\end{array}\right)+\sum_{i=0}^{j-k+1}(-1)^{i+1}\left(\begin{array}{l}
j \\
k
\end{array}\right)\left(\begin{array}{c}
j-k \\
i-1
\end{array}\right) \\
= & -\left(\begin{array}{c}
j \\
k-1
\end{array}\right) \sum_{i=0}^{j-k+1}(-1)^{i}\left(\begin{array}{c}
j-k+1 \\
i
\end{array}\right)-\left(\begin{array}{l}
j \\
k
\end{array}\right) \sum_{i=0}^{j-k+1}(-1)^{i}\left(\begin{array}{c}
j-k \\
i-1
\end{array}\right) \\
= & -\left(\begin{array}{c}
j \\
k-1
\end{array}\right) \sum_{i=0}^{j-k+1}(-1)^{i}\left(\begin{array}{c}
j-k+1 \\
i
\end{array}\right)+\left(\begin{array}{l}
j \\
k
\end{array}\right) \sum_{i=0}^{j-k}(-1)^{i}\left(\begin{array}{c}
j-k \\
i
\end{array}\right) .
\end{aligned}
$$

The first term is 0 unless $j=k-1$ and the second is 0 unless $j=k$. We easily check that the sum is -1 when $j=k-1$ and 1 when $j=k$, giving the result.

We are now ready to prove Theorem D.

Theorem 30. Suppose $\Delta$ is pure. Then

$$
\sum_{\substack{T \in \Delta \\|T|=k}} \tilde{\chi}\left(\mathrm{lk}_{\Delta}(T)\right)=\chi\left([\Delta]_{>k}\right)-\chi\left([\Delta]_{>k-1}\right)
$$

Proof. The claim is clear if $k=0$, since $[\Delta]_{>0}$ is the barycentric subdivision of $\Delta$, and since $[\Delta]_{>-1}$ is a cone. So we suppose $k \geqslant 1$. 
We have

$$
\begin{aligned}
& \sum_{T \in F_{k}} \tilde{\chi}\left(\mathrm{lk}_{\Delta}(T)\right) \\
& =\sum_{i=0}^{d-k} \sum_{T \in F_{k}}(-1)^{i+1} f_{i-1}\left(\mathrm{lk}_{\Delta}(T)\right) \\
& =\sum_{i=0}^{d-k}(-1)^{i+1}\left(\begin{array}{c}
i+k \\
k
\end{array}\right) f_{i+k-1}(\Delta) \\
& \left.=\sum_{i=0}^{d-k} \sum_{j=i+k-1}^{d-1}(-1)^{i+1}\left(\begin{array}{c}
i+k \\
k
\end{array}\right)\left(\begin{array}{c}
j \\
i+k-1
\end{array}\right) \chi\left(N_{j+1}(\Delta)\right) \quad(\text { By Theorem } 2.113)\right) \\
& =\sum_{j=0}^{d-1} \sum_{i=0}^{j}(-1)^{i+1}\left(\begin{array}{c}
i+k \\
k
\end{array}\right)\left(\begin{array}{c}
j \\
i+k-1
\end{array}\right) \chi\left(N_{j+1}(\Delta)\right) \\
& =\chi\left(N_{k+1}(\Delta)\right)-\chi\left(N_{k}(\Delta)\right)
\end{aligned}
$$

The result then follows from Theorem 14 (4).

Note that, as long as $k \neq d, \sum_{T \in F_{k}} \tilde{\chi}\left(\mathrm{lk}_{\Delta}(T)\right)=\chi\left([\Delta]_{>k}\right)-\chi\left([\Delta]_{>k-1}\right)=\widetilde{\chi}\left([\Delta]_{>k}\right)-$ $\tilde{\chi}\left([\Delta]_{>k-1}\right)$.

Remark 31. Examples 22, 38, and 39 demonstrate the sharpness of Theorem 30.

Corollary 32. Suppose $\Delta$ is pure. Then

$$
\sum_{k=j}^{i} \sum_{T \in F_{k}} \tilde{\chi}\left(\mathrm{lk}_{\Delta}(T)\right)=\chi\left([\Delta]_{>i}\right)-\chi\left([\Delta]_{>j-1}\right) .
$$

In particular,

$$
\sum_{k=0}^{i} \sum_{T \in F_{k}} \widetilde{\chi}\left(\mathrm{lk}_{\Delta}(T)\right)=\chi\left([\Delta]_{>i}\right) .
$$

As an application, we provide a result analogous to those of sections 3 and 4 for Gorenstein* complexes.

Corollary 33. Suppose $\Delta$ is Gorenstein*. Then

$$
\operatorname{dim}_{k} \widetilde{H}_{i-1}\left([\Delta]_{>j}\right)= \begin{cases}\operatorname{dim}_{k} \widetilde{H}_{j-1}\left(\Delta^{(j-1)}\right) & \text { if } i=d-j \\ 0 & \text { if } i \neq d-j\end{cases}
$$

The converse holds if $\operatorname{lk}_{\Delta}(T)$ is not acyclic for each $T \in \Delta$. 
Proof. By Theorem $14(4), \widetilde{H}_{i-1}\left([\Delta]_{>j}\right) \cong \widetilde{H}_{i-1}\left(N_{j+1}(\Delta)\right)$ for any $i$ and $j$. Thus, by Theorems 14 (1) and 9, both conditions imply $\Delta$ is Cohen-Macaulay, in particular, that $\Delta^{(j-1)}$ is Cohen-Macaulay for every $j$ ([Fr90, Theorem 8$\left.]\right)$. In this case, we have

$$
\operatorname{dim}_{k} \widetilde{H}_{j-1}\left(\Delta^{(j-1)}\right)=(-1)^{j} \widetilde{\chi}\left(\Delta^{(j-1)}\right)=\sum_{k=0}^{j}(-1)^{j-k} f_{k-1}(\Delta) .
$$

Suppose $\Delta$ is Gorenstein*. Then, by Theorem 9

$$
\widetilde{H}_{i-1}\left(\mathrm{lk}_{\Delta}(T)\right) \cong\left\{\begin{array}{ll}
k & \text { if } i=d-j \\
0 & \text { if } i \neq d-j
\end{array} .\right.
$$

Likewise, since $\Delta$ is Cohen-Macaulay, we have $\widetilde{H}_{i-1}\left([\Delta]_{>j}\right)=0$ unless $i=d-j$ by Theorem 14. We thus have

$$
\begin{aligned}
(-1)^{d-j-1} \operatorname{dim}_{k} \widetilde{H}_{d-j-1}\left([\Delta]_{>j}\right) & =\chi\left([\Delta]_{>j}\right) \\
& =\sum_{k=0}^{j} \sum_{T \in F_{k}} \widetilde{\chi}\left(\operatorname{lk}_{\Delta}(T)\right) \\
& =\sum_{k=0}^{j} \sum_{T \in F_{k}}(-1)^{d-k-1} \\
& =\sum_{k=0}^{j}(-1)^{d-k-1} f_{k-1}(\Delta) \\
& =(-1)^{d-j-1} \sum_{k=0}^{j}(-1)^{j-k} f_{k-1}(\Delta) \\
& =(-1)^{d-j-1} \operatorname{dim}_{k} \widetilde{H}_{j-1}\left(\Delta^{(j-1)}\right)
\end{aligned}
$$

and the claim follows.

Now suppose $\operatorname{lk}_{\Delta}(T)$ is non-acyclic for each $T \in \Delta$ and that

$$
\operatorname{dim}_{k} \widetilde{H}_{i-1}\left([\Delta]_{>j}\right)= \begin{cases}\sum_{k=0}^{j}(-1)^{j-k} f_{k-1}(\Delta) & \text { if } i=d-j \\ 0 & \text { if } i \neq d-j\end{cases}
$$

Since $\Delta$ is Cohen-Macaulay, $\widetilde{H}_{i-1}\left(\mathrm{lk}_{\Delta}(T)\right)=0$ unless $i=d-|T|$. Now we induct on $|T|$ to show that $\widetilde{H}_{d-|T|-1}\left(\operatorname{lk}_{\Delta}(T)\right) \cong k$ for each $T$. When $T=\varnothing$ we have $\operatorname{dim} \widetilde{H}_{d-1}\left(\operatorname{lk}_{\Delta} T\right)=$ $\operatorname{dim} \widetilde{H}_{d-1}(\Delta) \cong \operatorname{dim} \widetilde{H}_{d-1}\left([\Delta]_{>0}\right)=f_{-1}(\Delta)=1$. Now suppose $\widetilde{H}_{d-|T|-1}\left(\mathrm{lk}_{\Delta}(T)\right) \cong k$ whenever $|T|<j$. Then

$$
\sum_{k=0}^{j} \sum_{T \in F_{k}} \tilde{\chi}\left(\mathrm{lk}_{\Delta}(T)\right)=\tilde{\chi}\left([\Delta]_{>j}\right)
$$




$$
=(-1)^{d-j-1} \operatorname{dim}_{k} \widetilde{H}_{d-j-1}\left(N_{j+1}(\Delta)\right)=\sum_{k=0}^{j}(-1)^{d-k-1} f_{k-1}(\Delta)
$$

Similarly,

$$
\sum_{k=0}^{j-1} \sum_{T \in F_{k}} \tilde{\chi}\left(\mathrm{lk}_{\Delta}(T)\right)=\sum_{k=0}^{j-1}(-1)^{d-k-1} f_{k-1}(\Delta),
$$

and thus

$$
\sum_{T \in F_{j}} \tilde{\chi}\left(\mathrm{lk}_{\Delta}(T)\right)=\sum_{T \in F_{j}}(-1)^{d-j-1} \operatorname{dim}_{k} \widetilde{H}_{d-j-1}\left(\mathrm{lk}_{\Delta}(T)\right)=(-1)^{d-j-1} f_{j-1}(\Delta) .
$$

Then

$$
\sum_{T \in F_{j}} \operatorname{dim}_{k} \widetilde{H}_{d-j-1}\left(\mathrm{lk}_{\Delta}(T)\right)=f_{j-1}(\Delta)
$$

but, since $\operatorname{lk}_{\Delta}(T)$ is not acyclic for each $T$, we must have $\operatorname{dim}_{k} \widetilde{H}_{d-j-1}\left(\operatorname{lk}_{\Delta}(T)\right)=1$ for each $T \in F_{j}$, by the pigeonhole principle. The result now follows from induction.

Remark 34. We claim the result of Corollary 33 is analogous to those of Sections 3 and 4 , but this is perhaps not obvious. To see this, note that $\operatorname{dim}_{k} \widetilde{H}_{i-1}\left(\Delta^{(j-1)}\right)=$ $\operatorname{dim}_{k} \widetilde{H}_{i-1}\left(P_{>d-j}^{o p}\right)$ where $P$ is the face poset of $\Delta$ and $P^{o p}$ is its opposite poset. In essence, our result says that, when $\Delta$ is Gorenstein*, removing $j$ ranks from the bottom of $P$ gives the same homologies as removing $d-j$ ranks from the top, though these homologies are in different degrees.

\section{Open Problems and Examples}

In this section we provide examples illustrating the sharpness of the results from previous sections, and we discuss some related open questions. We begin by considering more general notions of independent and excellent sets. Through this section we continue to let $\Delta$ be a simplicial complex of dimension $d-1$. We say that $A \subseteq \Delta$ is independent if $\sigma \cup \tau \notin \Delta$ for all $\sigma, \tau \in A$ with $\sigma \neq \tau$. We say that $A$ is excellent if, additionally, for every facet $F$ of $\Delta, F \supseteq \sigma$ for some (necessarily unique) $\sigma \in A$. Note that $J=\left\{v_{1}, \ldots, v_{m}\right\} \subseteq V$ is independent (resp. excellent) if and only if $\left\{\left\{v_{1}\right\}, \ldots,\left\{v_{m}\right\}\right\}$ is an independent (resp. excellent) subset of $\Delta$. If $A \subseteq \Delta$ is independent, we set

$$
\Delta_{A}:=\Delta-\{\sigma \in \Delta \mid \sigma \supseteq \tau \text { for some } \tau \in A\} .
$$

If $A=\left\{\left\{v_{1}\right\}, \ldots,\left\{v_{m}\right\}\right\}$ where $J=\left\{v_{1}, \ldots, v_{m}\right\} \subseteq V$ is independent, then $\Delta_{A}=\operatorname{ast}_{\Delta}(J)$. Essentially the same argument as [Hib91, Proposition 2.8] shows the following extension of Lemma 24:

Proposition 35. Suppose $A \subseteq \Delta$ is independent. Then $\operatorname{depth} \Delta_{A} \geqslant \operatorname{depth} \Delta-1$.

We conjecture a similar extension of Lemma 15. 
Conjecture 36. Suppose $A \subseteq \Delta$ is excellent. If $\Delta$ satisfies $\left(S_{\ell}\right)$, then $\Delta_{A}$ satisfies $\left(S_{\ell}\right)$.

Remark 37. If $A$ is independent and $\ell \geqslant 2$, the conclusion can only hold if $A$ is excellent, since $\left(S_{2}\right)$ complexes are pure. Similar to Proposition 35, one can modify the argument of [Hib91, Proposition 2.8] to show that $\Delta_{A}$ satisfies $\left(S_{\ell-1}\right)$ whenever $\Delta$ satisfies $\left(S_{\ell}\right)$ and $A$ is excellent. However, as in the proof of Theorem 17, one often needs to cut away excellent subsets inductively, and, for this purpose, $\left(S_{\ell-1}\right)$ is not generally good enough; in particular, we cannot conclude anything when $\Delta$ only satisfies $\left(S_{2}\right)$. A positive answer to this conjecture would allow one to extend Theorem A to balanced complexes of a more general type, along the lines of [Hib91, Section 3].

The following examples show the converses of Theorems 17 and 18 do not hold, even for face posets of simplicial complexes:

Example 38. Consider the complex $\Delta_{1}$ with facets:

$$
\{4,5,6\},\{1,5,6\},\{1,3,5\},\{2,3,6\},\{2,5,6\},\{2,4,6\} \text {. }
$$

This complex is not $\left(S_{2}\right)$ but has $\widetilde{H}_{i-1}\left(\left[\Delta_{1}\right]_{>j}\right)=0$ for all $i, j$ with $i+j<d$ and $0 \leqslant i<2$.

Example 39. Consider the complex $\Delta_{2}$ with facets:

$$
\{4,5,6\},\{3,5,6\},\{2,3,5\},\{2,3,4\},\{1,3,4\},\{2,4,6\} .
$$

This complex is $\left(S_{2}\right)$ but $\widetilde{H}_{1}\left(\left[\Delta_{2}\right]_{>0}\right)$ is non-trivial.

In fact, $\widetilde{H}_{i-1}\left(\left[\Delta_{1}\right]_{>j}\right) \cong \widetilde{H}_{i-1}\left(\left[\Delta_{2}\right]_{>j}\right)$ for every $i$ and every $j$. Since $\Delta_{2}$ is $\left(S_{2}\right)$ and $\Delta_{1}$ is not, this shows that $\left(S_{2}\right)$ cannot be determined in general by reduced homologies of the $[\Delta]_{>j}$. Further, Example 39 is Buchsbaum while Example 38 is not, so Buchsbaum cannot be determined either. In a similar fashion, the following example shows that Gorenstein cannot be detected in general.

Example 40. Let $\Gamma_{1}$ be the complex with facets

$$
\{2,3,4\},\{1,3,4\},\{1,2,5\},\{2,3,5\},\{1,2,4\},\{1,3,5\}
$$

and $\Gamma_{2}$ the complex with facets

$$
\{1,2,3\},\{1,2,4\},\{1,3,4\},\{2,3,4\},\{1,2,5\},\{1,3,5\} .
$$

Then $\left[\Gamma_{1}\right]_{>j}$ and $\left[\Gamma_{2}\right]_{>j}$ have isomorphic homologies for each $j$, but $\Gamma_{1}$ is Gorenstein whilst $\Gamma_{2}$ is not (it is not even 2-Cohen-Macaulay).

The above discussion leads us to ask the following general question:

Question 41. In addition to the reduced homologies of the $[\Delta]_{>j}$, what information does one need to determine if a simplicial complex satisfies conditions such as $\left(S_{\ell}\right)$, Buchsbaum, or Gorenstein? 


\section{Acknowledgements}

We would like to thank Joseph Doolittle, Ken Duna, and Bennet Goeckner; we extend a special thanks to our advisor Hailong Dao and to Jonathan Montaño for providing useful feedback on earlier drafts on the paper. We would also like to thank Vic Reiner for helpful discussions, which led us to examine balanced simplicial complexes. We thank an anonymous referee whose careful reading and detailed feedback greatly improved the paper.

\section{References}

[BFS87] A. Björner, P. Frankl, and R. Stanley, The number of faces of balanced CohenMacaulay complexes and a generalized Macaulay theorem, Combinatorica 7 (1987), no. 1, 23-34. MR 905148

[BGS82] A. Björner, A. M. Garsia, and R. P. Stanley, An introduction to CohenMacaulay partially ordered sets, Ordered sets (Banff, Alta., 1981), NATO Adv. Study Inst. Ser. C: Math. Phys. Sci., vol. 83, Reidel, Dordrecht-Boston, Mass., 1982, pp. 583-615. MR 661307

[BH93] Winfried Bruns and Jürgen Herzog, Cohen-Macaulay rings, Cambridge Studies in Advanced Mathematics, vol. 39, Cambridge University Press, Cambridge, 1993. MR 1251956

[DDD $\left.{ }^{+} 19\right]$ Hailong Dao, Joseph Doolittle, Ken Duna, Bennet Goeckner, Brent Holmes, and Justin Lyle, Higher nerves of simplicial complexes, Algebr. Comb. 2 (2019), no. 5, 803-813. MR 4023567

[Fr90] Ralf Fröberg, On Stanley-Reisner rings, Topics in algebra, Part 2 (Warsaw, 1988), Banach Center Publ., vol. 26, PWN, Warsaw, 1990, pp. 57-70. MR 1171260

[Gar80] Adriano M. Garsia, Combinatorial methods in the theory of Cohen-Macaulay rings, Adv. in Math. 38 (1980), no. 3, 229-266. MR 597728

[Gib10] Peter Giblin, Graphs, surfaces and homology, third ed., Cambridge University Press, Cambridge, 2010. MR 2722281

[Hib91] Takayuki Hibi, Quotient algebras of Stanley-Reisner rings and local cohomology, J. Algebra 140 (1991), no. 2, 336-343. MR 1120426

[HN02] Patricia Hersh and Isabella Novik, A short simplicial h-vector and the upper bound theorem, Discrete Comput. Geom. 28 (2002), no. 3, 283-289. MR 1923232

[MT09] Satoshi Murai and Naoki Terai, h-vectors of simplicial complexes with Serre's conditions, Math. Res. Lett. 16 (2009), no. 6, 1015-1028. MR 2576690

[Mun84] James R. Munkres, Topological results in combinatorics, Michigan Math. J. 31 (1984), no. 1, 113-128. MR 736476 
[PSFTY14] M. R. Pournaki, S. A. Seyed Fakhari, N. Terai, and S. Yassemi, Survey article: Simplicial complexes satisfying Serre's condition: a survey with some new results, J. Commut. Algebra 6 (2014), no. 4, 455-483. MR 3294858

[Rei76] Gerald Allen Reisner, Cohen-Macaulay quotients of polynomial rings, Advances in Math. 21 (1976), no. 1, 30-49. MR 0407036

[Sta79] Richard P. Stanley, Balanced Cohen-Macaulay complexes, Trans. Amer. Math. Soc. 249 (1979), no. 1, 139-157. MR 526314

[Sta96] C Combinatorics and commutative algebra, second ed., Progress in Mathematics, vol. 41, Birkhäuser Boston, Inc., Boston, MA, 1996. MR 1453579

[Swa05] E. Swartz, Lower bounds for h-vectors of $k$-CM, independence, and broken circuit complexes, SIAM J. Discrete Math. 18 (2004/05), no. 3, 647-661. MR 2134424

[Ter07] Naoki Terai, Alexander duality in Stanley-Reisner rings, Affine algebraic geometry, Osaka Univ. Press, Osaka, 2007, pp. 449-462. MR 2330484

[Yan11] Kohji Yanagawa, Dualizing complex of the face ring of a simplicial poset, J. Pure Appl. Algebra 215 (2011), no. 9, 2231-2241. MR 2786613 